\title{
ARTICLES
}

\section{Localized-density-matrix implementation of time-dependent density-functional theory}

\author{
Chi Yung Yam, Satoshi Yokojima, ${ }^{\text {a) }}$ and GuanHua Chen ${ }^{\text {b) }}$ \\ Department of Chemistry, The University of Hong Kong, Pokfulam Road, Hong Kong
}

(Received 29 July 2003; accepted 6 August 2003)

\begin{abstract}
A linear-scaling first-principles quantum mechanical method is developed to evaluate the optical responses of large molecular systems. Instead of a many-body wave function, the equation of motion is solved for the reduced single-electron density matrix in the time domain. The locality of the reduced single-electron density matrix is utilized to ensure that computational time scales linearly with system size. The two-electron Coulomb integrals are evaluated with the fast multipole method, and the calculation of exchange-correlation quadratures utilizes the locality of an exchange-correlation functional and the integral prescreening technique. As an illustration, the resulting time-dependent density-functional theory is used to calculate the absorption spectra of polyacetylene oligomers and linear alkanes. The linear-scaling of computational time versus the system size is clearly demonstrated. (C) 2003 American Institute of Physics.
\end{abstract}

[DOI: $10.1063 / 1.1613634]$

\section{INTRODUCTION}

In recent years density-functional theory $(\mathrm{DFT})^{1-3}$ has become a promising alternative to conventional ab initio molecular orbital methods in quantum chemistry. The cost of a DFT calculation is on the same order as that of HartreeFock (HF), and with a description of electron correlation included, is substantially less expensive than traditional correlation techniques, such as the configuration interaction (CI), ${ }^{4}$ the Møller-Plesset perturbation theory (MP), ${ }^{5}$ and the coupled cluster $(\mathrm{CC})^{6}$ methods. DFT is founded upon the Hohenberg-Kohn theorem, ${ }^{1}$ which states that the exact ground-state energy is a unique functional of the exact electron density. The Kohn-Sham (KS) formulation ${ }^{2}$ of DFT, which is well suited for practical computation, is closely analogous to HF theory, in that a set of molecular orbitals is derived from an effective one-electron potential via a selfconsistent procedure. To solve for excited states or investigate properties involving time-dependent fields, the Hohenberg-Kohn-Sham theory has been generalized to treat time-dependent systems. It is based on the RungeGross theorem. ${ }^{7}$ Time-dependent density-functional theory (TDDFT) has become a powerful tool to calculate the excited state properties of molecules, such as polarizabilities, hyperpolarizabilities, and excited state energies. Two types of TDDFT formalisms exist. The first one relates directly to the linear response of electron density and leads to the density-based equation. This was suggested and employed $^{8-10}$ prior to the rigorous proof of the Runge-Gross theorem. The second formalism is based on the response of

\footnotetext{
${ }^{a}$ Current address: Institute of Materials Science, University of Tsukuba, 1-1-1 Ten-nodai, Tsukuba, Ibaraki 305-8573 Japan.

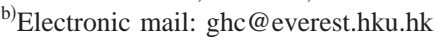

reduced density matrix, and thus leads to a density-matrixbased approach. ${ }^{11-13}$ TDDFT has been widely used to calculate the excited state properties of atoms and molecules. The state-of-the-art TDDFT calculations scale formally as $O\left(N^{3}\right),{ }^{11,14}$ where $N$ is the number of atoms involved. This makes TDDFT a relatively expensive numerical method, and cannot be employed to calculate the properties of very large molecules. It is thus desirable to have linear-scaling TDDFT whose computational time scales as $O(N)$.

Much progress has been made for linear-scaling DFT. ${ }^{15-22}$ The bottlenecks were the calculations of twoelectron Coulomb integrals and exchange-correlation (XC) quadratures, and the Hamiltonian diagonalization. The fast multipole method (FMM), ${ }^{23-26}$ which was originally developed to evaluate the Coulomb interactions of point charges, led to the linear-scaling computation of the two-electron Coulomb integrals. The linear-scaling evaluation of the XC quadratures was achieved by exploiting the localized nature of $\mathrm{XC}$ potential and by employing the integral prescreening technique. ${ }^{21,27,28}$ The Hamiltonian diagonalization is intrinsically $O\left(N^{3}\right)$, and most $O(N)$ algorithms make use of the locality or "nearsightedness" 18 of reduced single-electron density matrix $\rho$. In the divide-and-conquer (DAC) method, ${ }^{16,17} \rho$ is patched together from the pieces that are calculated for smaller subsystems, and this avoids the diagonalization of the Hamiltonian matrix of an entire system. Density-matrix-based energy minimization ${ }^{20,29}$ provides an alternative to the diagonalization, in which the energy is minimized upon the variation of $\rho$. Other linear-scaling methods have been developed as well, such as, the Fermi Operator Expansion (FOE) ${ }^{30}$ the Fermi Operator projection $(\mathrm{FOP}),{ }^{30}$ and the Orbital Minimization $(\mathrm{OM}) .^{31,32}$ 
These works pave the way for the linear-scaling TDDFT method.

The remaining obstacle for the linear-scaling TDDFT method lies in solving the TDDFT equation. The TDDFT equation is very similar to the time-dependent Hartree-Fock (TDHF) equation. ${ }^{33,34}$ The localized-density-matrix (LDM) method was developed to solve the TDHF equation, and its computational time scales linearly with the system size. ${ }^{35,36}$ Instead of the many-body wave function, the LDM method solves for reduced single-electron density matrix $\rho$ of a molecular system from which its electronic excited state properties are evaluated. The reduced single-electron density matrix $\rho$ contains important information of an electronic system, and its elements are defined as the expectation values, for instance, $\rho_{i j} \equiv\left\langle\Psi\left|a_{j}^{\dagger} a_{i}\right| \Psi\right\rangle$, with $\Psi$ being the many-body wave function and $a_{i}^{\dagger}\left(a_{j}\right)$ the electron creation (annihilation) operator at the local orbital $i(j)$. Expressed in an orthonormal basis set, the diagonal element $\rho_{i i}$ is the electron occupation number at a local orbital $i$, and the off-diagonal element $\rho_{i j}(i \neq j)$ measures the electronic coherence between two orthogonal local orbitals, $i$ and $j$. An equation of motion (EOM) for $\rho$ has been solved to calculate linear and nonlinear electronic responses to external fields, ${ }^{33,34}$ and thus, probe the properties of the excited states. This EOM is based on the TDHF approximation, ${ }^{33,34}$ and the computational time for solving it in the time domain scales as $O\left(N^{3}\right)$. In frequency domain, the computational time for solving it via diagonalization scales as $O\left(N^{6}\right)$ and the Davidson diagonalization ${ }^{37}$ can reduce the computational time substantially. It has been shown that the ground state off-diagonal elements $\rho_{i j}$ are negligible when the distance $r_{i j}$ between $i$ and $j$ is larger than a critical length $l_{0} \cdot{ }^{38}$ This is a consequence of "the nearsightedness of equilibrium systems." 18 When the system is subjected to an external field $\mathcal{E}(t)$, the field induces a change $\delta \rho$ in the reduced density matrix. The induced density matrix $\delta \rho$ has a similar "nearsightedness," i.e., off-diagonal element $\delta \rho_{i j}$ is approximately zero, as the distance between $i$ and $j$ is large enough. ${ }^{38}$ Different orders of responses in $\mathcal{E}(t)$ have different critical lengths. Usually the higher the order of response $n$ is, the longer the critical length $l_{n}$ is, i.e., $l_{0} \leqslant l_{1} \leqslant l_{2} \leqslant l_{3} \leqslant \cdots$. We may truncate the $n$ th-order-induced density matrix $\delta \rho^{(n)}$ (note that $\delta \rho=\delta \rho^{(1)}+\delta \rho^{(2)}+\delta \rho^{(3)}+\cdots$ ) by setting its elements $\delta \rho_{i j}^{(n)}$ to zero if $r_{i j}>l_{n}$. This truncation leads to a drastic reduction of the computational time. Since TDDFT and TDHF have similar EOMs for $\rho$, we may combine the TDDFT and LDM methods just as TDHF-LDM method. $^{35}$ The computational time of the resulting TDDFT-LDM method should thus scale linearly with the system size.

In this manuscript we present the TDDFT-LDM method for calculating the excited state properties of very large molecular systems and its applications to polyacetylene oligomers and alkanes. In Sec. II we outline the TDDFT method, and, in particular, its local density approximation (TDLDA) approach. The TDLDA method is combined with the LDM method, and the resulting TDLDA-LDM formalism is presented. To test its validity, we apply the TDLDA-LDM method to calculate the absorption spectra of alkanes and polyenes. The calculation details and results are presented in Sec. III. We discuss the further development of TDDFT-LDM method and conclude in Sec. IV.

\section{COMBINED TIME-DEPENDENT DENSITY FUNCTIONAL THEORY AND LOCALIZED-DENSITY- MATRIX METHOD}

\section{A. Time-dependent local density approximation}

When an external electromagnetic field is applied to a molecule, its electronic response to the field $\mathcal{E}(t)$, and optical signals may be observed. The EOM for $\rho$ has been derived within the TDHF approximation. ${ }^{34}$ For TDDFT, the EOM can be derived in the similar way. Starting with the definition of reduced single-electron density matrix $\rho\left(\mathbf{r}, \mathbf{r}^{\prime}, t\right)$ in the spatial representation,

$$
\rho\left(\mathbf{r}, \mathbf{r}^{\prime}, t\right)=\sum_{k}^{\text {occ }} \psi_{k}^{*}\left(\mathbf{r}^{\prime}, t\right) \psi_{k}(\mathbf{r}, t),
$$

where $\psi_{k}(\mathbf{r}, t)$ is the $k$ th occupied molecular orbital (MO) and the summation is over all occupied MOs. The reduced single-electron density matrix $\rho$ in the atomic orbital (AO) basis set is given by

$$
\rho_{i j}(t)=\int d \mathbf{r} \int d \mathbf{r}^{\prime} \phi_{i}^{*}\left(\mathbf{r}^{\prime}\right) \rho\left(\mathbf{r}, \mathbf{r}^{\prime}, t\right) \phi_{j}(\mathbf{r}),
$$

where $\phi_{i}(\mathbf{r})$ is the $i$ th atomic orbital.

From the time-dependent Schrödinger equation,

$$
\begin{aligned}
& i \hbar \frac{\partial}{\partial t} \psi_{i}(\mathbf{r}, t)=\hat{F}(n(\mathbf{r}, t), \mathbf{r}, t) \psi_{i}(\mathbf{r}, t), \\
& \hat{F}=\hat{h}(t)+\hat{f}(t), \\
& \hat{h}(t)=-\frac{1}{2} \nabla^{2}+U(\mathbf{r})+\int d \mathbf{r}^{\prime} \frac{n\left(\mathbf{r}^{\prime}, t\right)}{\left|\mathbf{r}-\mathbf{r}^{\prime}\right|}+v^{\mathrm{xc}}(\mathbf{r}, t), \\
& \hat{f}(t)=e \mathcal{E}(t) \cdot \mathbf{r},
\end{aligned}
$$

where $n(\mathbf{r}, t)$ is the electron density. $\hat{h}(t)$ is the Fock operator and $\hat{f}(t)$ is the interaction between an electron and the external field $\mathcal{E}(t)$. The first and second terms in Eq. (5) are the electron kinetic energy and the potential energy produced by the nuclei, respectively. The third terms in Eq. (5) represents the Coulomb interaction among the electrons and the fourth term, $v^{\mathrm{xc}}(\mathbf{r}, t)$ is the exchange-correlation interaction within the local density approximation (LDA).

The time derivative of Eq. (1) may be expressed as

$$
\begin{aligned}
i \hbar \frac{\partial}{\partial t} \rho\left(\mathbf{r}, \mathbf{r}^{\prime}, t\right)= & \sum_{k}^{\text {occ }} \hat{F} \psi_{k}(\mathbf{r}, t) \psi_{k}^{*}\left(\mathbf{r}^{\prime}, t\right) \\
& -\sum_{k}^{\text {occ }} \psi_{k}(\mathbf{r}, t)\left(\hat{F} \psi_{k}\left(\mathbf{r}^{\prime}, t\right)\right)^{*} .
\end{aligned}
$$

The EOM for the reduced single-electron density matrix $\rho$ in the $\mathrm{AO}$ representation can be thus expressed as 


$$
\begin{aligned}
i \hbar \frac{\partial}{\partial t} \rho_{i j}(t)= & \sum_{k}^{\text {occ }}\left\langle\phi_{i}|\hat{F}| \psi_{k}(\mathbf{r}, t)\right\rangle\left\langle\psi_{k}\left(\mathbf{r}^{\prime}, t\right) \mid \phi_{j}\right\rangle \\
& -\sum_{k}^{\text {occ }}\left\langle\phi_{i} \mid \psi_{k}(\mathbf{r}, t)\right\rangle\left\langle\psi_{k}\left(\mathbf{r}^{\prime}, t\right)|\hat{F}| \phi_{j}\right\rangle \\
= & \sum_{k}^{\text {occ }} \sum_{m n}\left\langle\phi_{i}|\hat{F}| \phi_{m}\right\rangle c_{m k}(t) c_{n k}^{*}(t)\left\langle\phi_{n} \mid \phi_{j}\right\rangle \\
& -\sum_{k}^{\text {occ }} \sum_{m n}\left\langle\phi_{i} \mid \phi_{m}\right\rangle c_{m k}(t) c_{n k}^{*}(t)\left\langle\phi_{n}|\hat{F}| \phi_{j}\right\rangle \\
= & \sum_{m n}\left(F_{i m} \rho_{m n} \delta_{n j}-\delta_{i m} \rho_{m n} F_{n j}\right) \\
= & \sum_{m}\left(F_{i m} \rho_{m j}-\rho_{i m} F_{m j}\right) .
\end{aligned}
$$

Here we assume the AOs are orthonormal. For a nonorthogonal basis set, a similar EOM can be derived with the inclusion of overlap matrix $S .{ }^{39}$

Within the TDDFT formalism, a closed nonlinear selfconsistent EOM is yielded for the reduced single-electron density matrix $\rho(t)$,

$$
i \hbar \dot{\rho}(t)=[h(t)+f(t), \rho(t)],
$$

where $h(t)$ is the Fock matrix,

$$
\begin{aligned}
& h_{m n}(t)=t_{m n}+v_{m n}^{\mathrm{xc}}(t)+\sum_{i j} \rho_{i j}(t) V_{m n i j}, \\
& t_{m n}=\left\langle\phi_{m}\left|-\frac{1}{2} \nabla^{2}+U(\mathbf{r})\right| \phi_{n}\right\rangle \\
& \quad=\int d \mathbf{r} \phi_{m}^{*}(\mathbf{r})\left[-\frac{1}{2} \nabla^{2}+U(\mathbf{r})\right] \phi_{n}(\mathbf{r}), \\
& v_{m n}^{\mathrm{xc}}(t)=\int d \mathbf{r} \phi_{m}^{*}(\mathbf{r}) v^{\mathrm{xc}}[n](\mathbf{r}, t) \phi_{n}(\mathbf{r}), \\
& V_{m n i j}=\int d \mathbf{r} \int d \mathbf{r}^{\prime} \phi_{m}^{*}(\mathbf{r}) \phi_{n}(\mathbf{r}) \frac{1}{\left|\mathbf{r}-\mathbf{r}^{\prime}\right|} \phi_{i}^{*}\left(\mathbf{r}^{\prime}\right) \phi_{j}\left(\mathbf{r}^{\prime}\right),
\end{aligned}
$$

with $t_{m n}$ being the one-electron integral element between orbitals $m$ and $n, V_{m n i j}$ the two-electron Coulomb integral, and $v^{\mathrm{xc}}[n](\mathbf{r}, t)$ is the exchange-correlation potential, which is defined as the functional derivative of the exchange correlation functional $A^{\mathrm{xc}}$,

$$
v^{\mathrm{xc}}[n](\mathbf{r}, t) \equiv \frac{\delta A^{\mathrm{xc}}[n]}{\delta n(\mathbf{r}, t)} \approx \frac{\delta E^{\mathrm{xc}}\left[n_{t}\right]}{\delta n_{t}(\mathbf{r})}=v^{\mathrm{xc}}\left[n_{t}\right](\mathbf{r}),
$$

where $n_{t}$ denotes the electron density evaluated at the time $t$, the unknown functional $A^{\mathrm{xc}}$ is approximated by $E^{\mathrm{xc}}$, which is the exchange-correlation functional of time-independent Kohn-Sham theory, and $E^{\mathrm{xc}}$ is further approximated by the local density approximation (LDA). ${ }^{2,340}$ Note that $A^{\mathrm{xc}}$ is a functional of a function $n$ over both time and space and $E^{\mathrm{xc}}$ is a functional of a function $n_{t}$ over space only. This is referred to as the adiabatic approximation in which the static LDA functional evaluated at the time-dependent density is used for $v^{\mathrm{xc}}[n](\mathbf{r}, t)$. The matrix elements of $f(t)$ are evaluated as

$$
f_{m n}(t)=e \mathcal{E}(t) \cdot\left\langle\phi_{m}|\hat{\mathbf{r}}| \phi_{n}\right\rangle .
$$

We partition the reduced single-electron density matrix $\rho(t)$ into two parts:

$$
\rho(t)=\rho^{(0)}+\delta \rho(t),
$$

where $\rho^{(0)}$ is the DFT ground state reduced single-electron density matrix in the absence of the external field, and $\delta \rho(t)$ is the difference between $\rho(t)$ and $\rho^{(0)}$, i.e., the induced reduced single-electron density matrix by $\mathcal{E}(t)$. Similarly, the Fock matrix $h(t)$ is decomposed in the form

$$
h(t)=h^{(0)}+\delta h(t),
$$

where $h^{(0)}$ is the Fock matrix when $\mathcal{E}(t)=0$ and $\delta h(t)$ is the external field induced Fock matrix,

$$
\delta h_{i j}(t)=\delta v_{i j}^{\mathrm{xc}}+\sum_{m n} \delta \rho_{m n}(t) V_{i j m n},
$$

where

$$
\begin{aligned}
\delta v_{m n}^{\mathrm{xc}}= & \int d \mathbf{r} \phi_{m}^{*}(\mathbf{r}) \delta v^{\mathrm{xc}}\left[n_{t}\right] \phi_{n}(\mathbf{r}) \\
= & \int d \mathbf{r} \int d \mathbf{r}^{\prime} \phi_{m}^{*}(\mathbf{r}) \phi_{n}(\mathbf{r}) \frac{\delta v^{\mathrm{xc}}\left[n_{t}\right](\mathbf{r})}{\delta n_{t}\left(\mathbf{r}^{\prime}\right)} \delta n_{t}\left(\mathbf{r}^{\prime}\right) \\
= & \int d \mathbf{r} \int d \mathbf{r}^{\prime} \phi_{m}^{*}(\mathbf{r}) \phi_{n}(\mathbf{r}) \frac{\delta v^{\mathrm{xc}}\left[n_{t}\right](\mathbf{r})}{\delta n_{t}\left(\mathbf{r}^{\prime}\right)} \\
& \times \sum_{i j} \phi_{i}\left(\mathbf{r}^{\prime}\right) \delta \rho_{i j} \phi_{j}^{*}\left(\mathbf{r}^{\prime}\right) \\
= & \sum_{i j} \delta \rho_{i j}(t) V_{m n i j}^{\mathrm{xc}}, \\
V_{m n i j}^{\mathrm{xc}} \equiv & \int d \mathbf{r} \int d \mathbf{r}^{\prime} \phi_{m}^{*}(\mathbf{r}) \phi_{n}(\mathbf{r}) \\
& \times \frac{\delta^{2} E^{\mathrm{xc}}[n]}{\delta n(\mathbf{r}) \delta n\left(\mathbf{r}^{\prime}\right)} \phi_{i}^{*}\left(\mathbf{r}^{\prime}\right) \phi_{j}\left(\mathbf{r}^{\prime}\right) \\
= & \int d \mathbf{r} \phi_{m}^{*}(\mathbf{r}) \phi_{n}(\mathbf{r}) \frac{\delta v^{\mathrm{xc}}\left[n_{t}\right](\mathbf{r})}{\delta n(\mathbf{r})} \phi_{i}^{*}(\mathbf{r}) \phi_{j}(\mathbf{r}),
\end{aligned}
$$

and, thus,

$$
\delta h_{i j}(t)=\sum_{m n} \delta \rho_{m n}(t)\left(V_{i j m n}+V_{i j m n}^{\mathrm{xc}}\right) .
$$

With Eqs. (16) and (17), we can rewrite Eq. (9) as

$i \hbar \delta \dot{\rho}=\left[h^{(0)}, \delta \rho\right]+\left[\delta h, \rho^{(0)}\right]+\left[f, \rho^{(0)}\right]+[f, \delta \rho]+[\delta h, \delta \rho]$.

Equation (21) is the EOM for $\delta \rho$ under the TDLDA approximation. To the first order in $\mathcal{E}(t)$, the EOM for the first-order-induced reduced single-electron density matrix $\delta \rho^{(1)}$ is expressed as

$$
i \hbar \delta \dot{\rho}^{(1)}=\left[h^{(0)}, \delta \rho^{(1)}\right]+\left[\delta h^{(1)}, \rho^{(0)}\right]+\left[f, \rho^{(0)}\right] .
$$

More specifically, 


$$
\begin{aligned}
i \hbar \delta \dot{\rho}_{i j}^{(1)}= & \sum_{k}\left(h_{i k}^{(0)} \delta \rho_{k j}^{(1)}-\delta \rho_{i k}^{(1)} h_{k j}^{(0)}\right) \\
& +\sum_{k}\left(\delta h_{i k}^{(1)} \rho_{k j}^{(0)}-\rho_{i k}^{(0)} \delta h_{k j}^{(1)}\right) \\
& +\sum_{k}\left(f_{i k} \rho_{k j}^{(0)}-\rho_{i k}^{(0)} f_{k j}\right),
\end{aligned}
$$

where the first-order induced Fock matrix element,

$$
\delta h_{i j}^{(1)}=\sum_{m n} \delta \rho_{m n}^{(1)}\left(V_{i j m n}+V_{i j m n}^{\mathrm{xc}}\right) .
$$

We integrate Eq. (23) numerically in the time domain and solve for the time evolution of the polarization vector $\mathbf{P}(t)$. Within the dipole approximation, $\mathbf{P}(t)$ may be expressed as

$$
\mathbf{P}(t)=-\sum_{i j} e\left\langle\phi_{i}|\hat{\mathbf{r}}| \phi_{j}\right\rangle \rho_{i j}(t) .
$$

The first-order response $\mathbf{P}^{(1)}(t)$ is

$$
\mathbf{P}^{(1)}(t)=-\sum_{i j} e\left\langle\phi_{i}|\hat{\mathbf{r}}| \phi_{j}\right\rangle \delta \rho_{i j}^{(1)}(t) .
$$

To obtain the optical absorption spectrum, we perform a Fourier transformation of $\mathbf{P}^{(1)}(t)$,

$$
\mathbf{P}^{(1)}(\omega)=\int_{-\infty}^{\infty} d t \mathbf{P}^{(1)}(t) e^{-i \omega t} .
$$

The ratio between the imaginary part of $\mathbf{P}^{(1)}(\omega)$ and $\mathcal{E}(\omega)$, i.e., $\operatorname{Im}\left[\mathbf{P}^{(1)}(\omega) / \mathcal{E}(\omega)\right]$, is the absorption amplitude at frequency $\omega$ where $\mathcal{E}(\omega)$ is the Fourier transform of $\mathcal{E}(t)$.

\section{B. Localized-density-matrix approximation and FMM evaluation of Coulomb interaction}

The key for the $O(N)$ scaling lies in the reduction of the dimension of the reduced single-electron density matrix. This reduction is based on the fact that the density matrix has a localized character or a "nearsightedness." 18 This "nearsightedness" holds not only for the $\rho^{(0)}$ but also for $\delta \rho .^{38}$ Specifically, $\rho_{i j}^{(0)}$ is set to zero for $r_{i j}>l_{0}$, and, consequently, $h_{i j}^{(0)}$ becomes zero for the same $r_{i j}$ [see Eq. (10)]; and $\delta \rho_{i j}^{(1)}$ is set to zero when $r_{i j}>l_{1}$, which leads to a reduction of the dimension of $\delta \rho^{(1)}$ from $O\left(N^{2}\right)$ to $O(N)::^{35,36}$

$$
\begin{aligned}
& \rho_{i j}^{(0)}=0, \quad \text { if } r_{i j}>l_{0} ; \\
& \delta \rho_{i j}^{(1)}=0, \quad \text { if } r_{i j}>l_{1} .
\end{aligned}
$$

Here $l_{0}$ and $l_{1}$ are two cutoff lengths. For a fixed pair of $i$ and $j$, these approximations result in the finite number of summations in Eq. (23) for $k$ on the right-hand side (rhs), which leads to $O(N)$ floating point operations for evaluation of the first and third terms on the rhs of Eq. (23). The second term on the rhs of Eq. (23) can be expanded as

$$
\begin{aligned}
\sum_{k} & \sum_{m} \sum_{n}\left(\delta \rho_{m n}^{(1)} V_{i k m n} \rho_{k j}^{(0)}-\rho_{i k}^{(0)} \delta \rho_{m n}^{(1)} V_{k j m n}\right) \\
& +\sum_{k} \sum_{m} \sum_{n}\left(\delta \rho_{m n}^{(1)} V_{i k m n}^{\mathrm{xc}} \rho_{k j}^{(0)}-\rho_{i k}^{(0)} \delta \rho_{m n}^{(1)} V_{k j m n}^{\mathrm{xc}}\right) .
\end{aligned}
$$

Although the number of summations over $k$ is finite for each pair of $i$ and $j$, the number of summations over $m$ and $n$ is $O(N)$. This leads to an overall $O\left(N^{2}\right)$ floating point operations for the second term on the rhs of Eq. (23). We separate the induced Fock matrix $\delta h^{(1)}$ into

$$
\begin{aligned}
& \delta h^{(1)}=\delta h^{u}+\delta h^{\mathrm{xc}}, \\
& \delta h_{i j}^{u}=\sum_{m n} \delta \rho_{m n}^{(1)} V_{i j m n}, \\
& \delta h_{i j}^{\mathrm{xc}}=\sum_{m n} \delta \rho_{m n}^{(1)} V_{i j m n}^{\mathrm{xc}} .
\end{aligned}
$$

Equation (29) can thus be rewritten as

$$
\sum_{k}\left(\delta h_{i k}^{u} \rho_{k j}^{(0)}-\rho_{i k}^{(0)} \delta h_{k j}^{u}\right)+\sum_{k}\left(\delta h_{i k}^{\mathrm{xc}} \rho_{k j}^{(0)}-\rho_{i k}^{(0)} \delta h_{k j}^{\mathrm{xc}}\right) .
$$

Due to the slow decaying nature of $1 / r$ in the Coulomb potential, one cannot simply cut off individual interactions below a certain threshold. We have to include all the pairwise interactions between these charge distributions. This yields $O\left(N^{2}\right)$ floating point operations to evaluate the first term of Eq. (33). To achieve a linear-scaling calculation of the first term of Eq. (33), we resort to the $\mathrm{FMM}^{23-26}$ to evaluate $\delta h_{i j}^{u}$. In the calculation, $\delta h^{u}$ and $\delta h^{\mathrm{xc}}$ are first calculated in the nonorthogonal basis set and then transformed to the orthogonal basis set.

For $\delta h^{u}$, it contains the Coulomb interaction between the charge distributions and the induced charge distributions,

$$
\begin{aligned}
& \delta h_{i j}^{u}=\int d \mathbf{r} \int d \mathbf{r}^{\prime} d_{i j}(\mathbf{r}) \frac{1}{\left|\mathbf{r}-\mathbf{r}^{\prime}\right|} \sum_{m n} \delta d_{m n}\left(\mathbf{r}^{\prime}\right), \\
& d_{i j}(\mathbf{r})=\phi_{i}^{*}(\mathbf{r}) \phi_{j}(\mathbf{r}), \\
& \delta d_{m n}\left(\mathbf{r}^{\prime}\right)=\delta \rho_{m n} \phi_{m}^{*}\left(\mathbf{r}^{\prime}\right) \phi_{n}\left(\mathbf{r}^{\prime}\right) .
\end{aligned}
$$

Here we define $d_{i j}$ and $\delta d_{i j}$ as the charge distribution and the induced charge distribution for a pair of atomic orbitals $i$ and $j$, respectively. Note that the numbers of $d_{i j}$ and $\delta d_{i j}$ are $O(N)$ since the overlap between $i$ and $j$ decays exponentially over their distance. For instance, the product of two $1 \mathrm{~s}$ Gaussian basis functions is expressed as ${ }^{41}$

$$
\begin{aligned}
& G_{1 s}^{\alpha}\left(\mathbf{r}-\mathbf{R}_{i}\right) G_{1 s}^{\beta}\left(\mathbf{r}-\mathbf{R}_{j}\right)= \exp \left[-\frac{\alpha \beta}{\alpha+\beta}\left|\mathbf{R}_{i}-\mathbf{R}_{j}\right|^{2}\right] \\
& \times G_{1 s}^{\alpha+\beta}\left(\mathbf{r}-\mathbf{R}_{P}\right), \\
& G_{1 s}^{\alpha}(\mathbf{r}-\mathbf{R})=\exp \left[-\alpha(\mathbf{r}-\mathbf{R})^{2}\right],
\end{aligned}
$$

where $\mathbf{R}_{i}$ and $\mathbf{R}_{j}$ are the centers of orbital $i$ and $j$, respectively, $\alpha$ and $\beta$ are the exponents, and

$$
\mathbf{R}_{P}=\frac{\alpha \mathbf{R}_{i}+\beta \mathbf{R}_{j}}{\alpha+\beta},
$$

is defined as the center of the product of the Gaussian basis functions.

Figure 1 shows the Coulomb interaction between the charge distributions, $d_{i j}$ and $\delta d_{m n}$. The whole physical space with all the charges and induced charges are contained in a large cube. The cube is then divided in half along each 


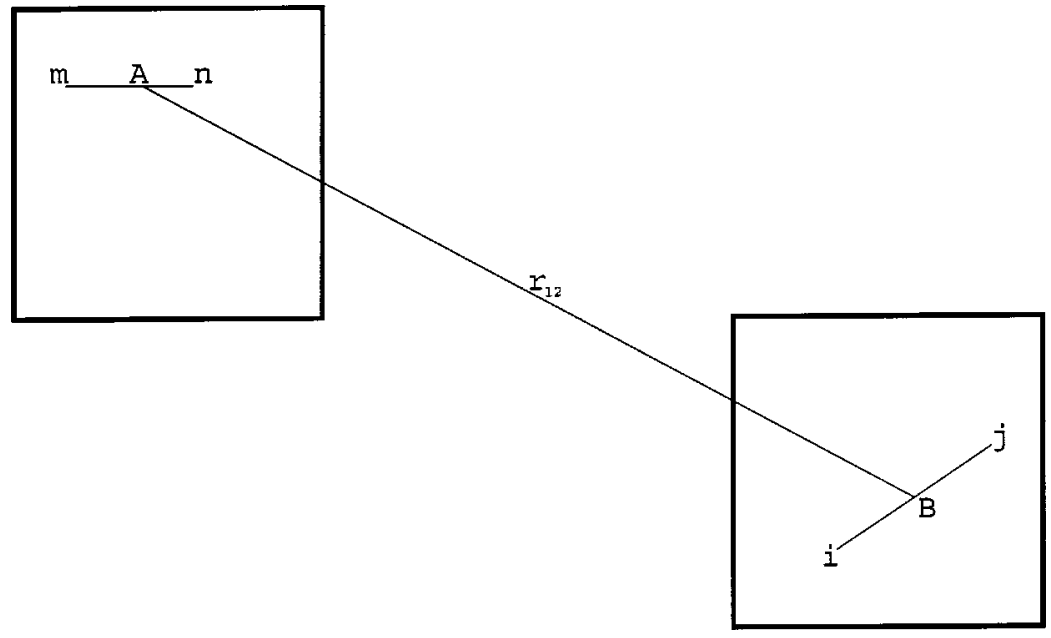

FIG. 1. Coulomb interaction between charge distribution $d_{i j}$ and induced charge distribution $\delta d_{m n} . A$ and $B$ are the center of charge $i, j$ and induced charge $m, n$, respectively.
Cartesian axis and thus each cube contains eight children cubes. This process is continued recursively until the charges in each cube at the lowest level is approximately constant. Figure 2 shows the first three levels of division. We evaluate the Coulomb potential at $P$ due to the induced charges in cube $A$, assuming $|\mathbf{R}| \gg\left|\mathbf{r}_{m n}\right|$ applies for all of the induced charges within the cube $A$ (cf. Fig. 3). The Coulomb potential can be expressed as the multipole expansion,

$$
\begin{aligned}
& V(\mathbf{r})=\frac{Z}{R}+\frac{\mu_{\alpha} R_{\alpha}}{R^{3}}+\frac{Q_{\alpha \beta} R_{\alpha} R_{\beta}}{R^{5}}+\frac{O_{\alpha \beta \gamma} R_{\alpha} R_{\beta} R_{\gamma}}{R^{7}}+\cdots, \\
& Z=\sum_{m n \in A} S_{m n} \delta \rho_{m n}^{\mathrm{AO}},
\end{aligned}
$$

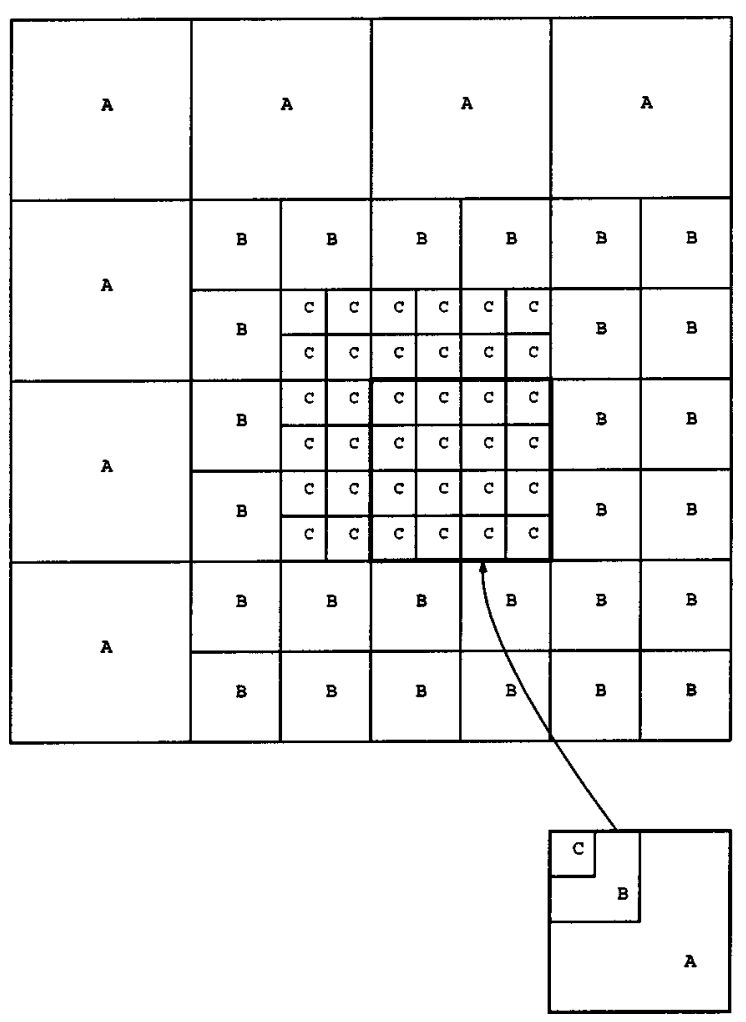

FIG. 2. Hierarchy of cubes.

$$
\begin{aligned}
& \mu_{\alpha}=\sum_{m n \in A}\left\langle\chi_{m}\left|r_{m n \alpha}\right| \chi_{n}\right\rangle \delta \rho_{m n}^{\mathrm{AO}}, \\
& Q_{\alpha \beta}=\sum_{m n \in A}\left\langle\chi_{m}\left|\frac{3}{2} r_{m n \alpha} r_{m n \beta}-\frac{1}{2} \delta_{\alpha \beta} r_{m n}^{2}\right| \chi_{n}\right\rangle \delta \rho_{m n}^{\mathrm{AO}}, \\
& O_{\alpha \beta \gamma}=\sum_{m n \in A}\left\langle\chi_{m}\right| \frac{15}{6} r_{m n \alpha} r_{m n \beta} r_{m n \gamma}-\frac{1}{2} r_{m n}^{2}\left(r_{m n \alpha} \delta_{\beta \gamma}\right. \\
& \left.\quad+r_{m n \beta} \delta_{\alpha \gamma}+r_{m n \gamma} \delta_{\alpha \beta}\right)\left|\chi_{n}\right\rangle \delta \rho_{m n}^{\mathrm{AO}},
\end{aligned}
$$

where $\mathbf{R}=\mathbf{r}-\mathbf{r}_{\mathbf{A}}, \mathbf{r}_{\mathbf{A}}$ is the center of the cube $A, \mathbf{r}$ is any point outside the cube, $\alpha=x, y, z, r_{m n \alpha}$ is the $\alpha$ component of the position vector for $\delta d_{m n}$ measured with respect to the center of the cube $A$, and $S_{m n}=\int d \mathbf{r} \chi_{m}^{*}(\mathbf{r}) \chi_{n}(\mathbf{r}) . \chi_{n}$ is the nonorthogonal atomic orbital $n$, and $\delta \rho^{\mathrm{AO}}$ is the induced density matrix in a nonorthogonal basis set. The multipole expansions of the potential caused by the induced charges of a cube at level $(l-1)$ can be constructed from those multipole expansions of the cubes at the lower level $(l)$ as follows:

$$
\begin{aligned}
Z^{(l-1)} & =\sum_{m n \in A_{l-1}} S_{m n} \delta \rho_{m n}^{\mathrm{AO}} \\
& =\sum_{A_{l} \in A_{l-1}} \sum_{m n \in A_{l}} S_{m n} \delta \rho_{m n}^{\mathrm{AO}}=\sum_{A_{l} \in A_{l-1}} Z^{(l)}, \\
\mu_{\alpha}^{(l-1)} & =\sum_{m n \in A_{l-1}}\left\langle\chi_{m}\left|r_{m n \alpha}^{(l-1)}\right| \chi_{n}\right\rangle \delta \rho_{m n}^{\mathrm{AO}} \\
& =\sum_{A_{l} \in A_{l-1}} \sum_{m n \in A_{l}}\left\langle\chi_{m}\left|r_{p c \alpha}^{(l)}+r_{m n \alpha}^{(l)}\right| \chi_{n}\right\rangle \delta \rho_{m n}^{\mathrm{AO}} \\
& =\sum_{A_{l} \in A_{l-1}} Z^{(l)} r_{p c \alpha}^{(l)}+\sum_{A_{l} \in A_{l-1}} \mu_{\alpha}^{(l)},
\end{aligned}
$$

etc., where $r_{p c \alpha}^{(l)}$ is the $\alpha$ component of the position vector for a child cube $(l)$ with respect to the center of its parent cube $(l-1) . A_{l}$ is defined as cube $A$ at level $l$. The multipole moments from all child cubes are summed and stored for the parent cube. Figure 4 shows the schematic relation between a parent cube and a child cube. At this point, we have a multipole expansion for each cube representing all the charge 


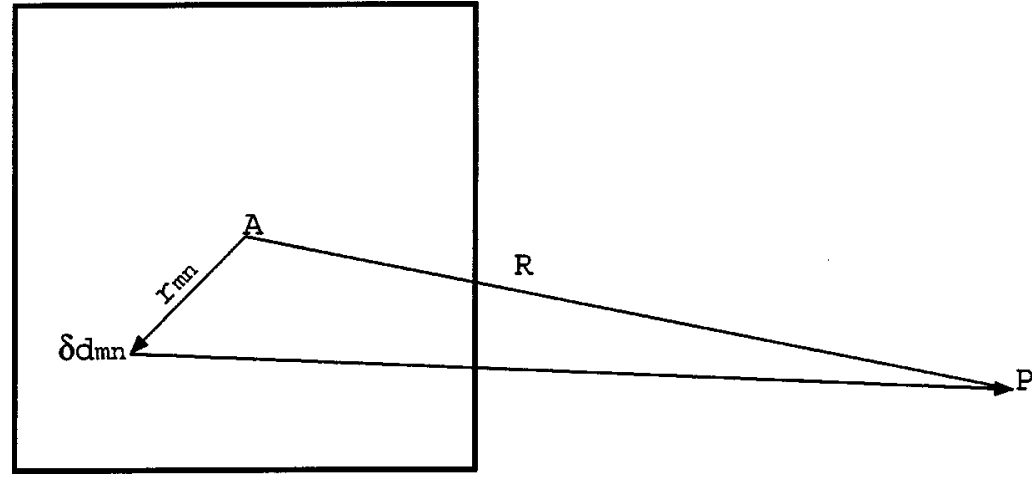

FIG. 3. Coulomb potential formed by $\delta d_{m n}$ on the point $P$.

distributions contained in it. Since the number of cubes scales linearly with the system size and the number of charge distributions in the lowest level is chosen to be independent of the system size, the CPU time for evaluating the multipole moments for cubes at different levels should therefore scale linearly.

The induced Coulomb potential inside a cube $B$ at the lowest level is partitioned into the near-field and farfield contributions $^{24}$ as

$$
\begin{aligned}
V(\mathbf{r}) & =V_{\text {far }}(\mathbf{r})+V_{\text {near }}(\mathbf{r}) \\
& =\sum_{A \in \text { far }} V\left(\mathbf{r}-\mathbf{r}_{A B}\right)+\sum_{m n \in \text { near }} \int d \mathbf{r}^{\prime} \frac{\chi_{m}^{*}\left(\mathbf{r}^{\prime}\right) \chi_{n}\left(\mathbf{r}^{\prime}\right) \delta \rho_{m n}^{\mathrm{AO}}}{\left|\mathbf{r}-\mathbf{r}^{\prime}\right|},
\end{aligned}
$$

where $\mathbf{r}_{A B}$ is defined as the displacement vector of the center of cube $A$ with respect to the center of $B$ and $\mathbf{r}$ is the charge position with respect to the center of $B$. If their center-tocenter distance is less than $a_{l} D$ along the $x, y$, and $z$ directions, where $a_{l}$ is the size of a cube at level $l$ and $D$ is a positive integer, then cube $A$ and $B$ are said to be the nearfield cubes, otherwise, they are considered as farfield cubes. For the near-field contribution where $d$ and $\delta d$ are close in distance, explicit analytical integration is used to ensure high accuracy. The computational time of the near-field interaction between $d$ and $\delta d$ is of $O(N M)$, where $M$ is the number of induced charges $\delta d_{m n}$ 's within the nearby cubes at the

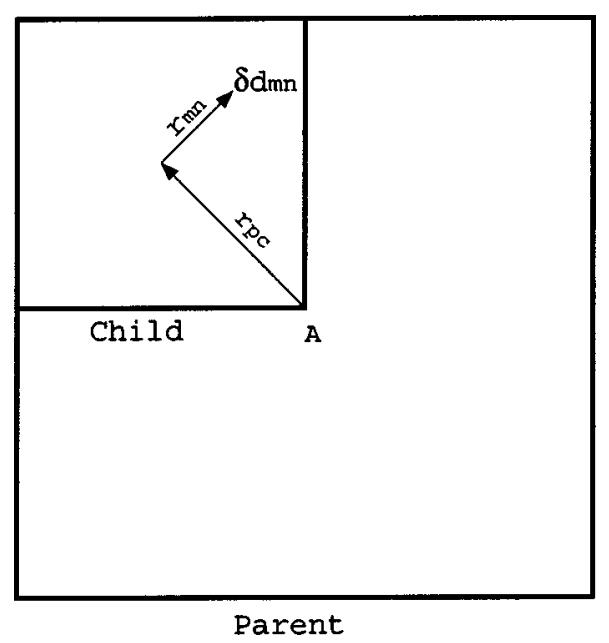

FIG. 4. The connection between the parent cube and his child. lowest level, and $N$ is the number of charges $d_{i j}$ 's of the entire system. The number of induced charges $\delta d_{m n}$ 's within each lowest level cube is independent of the system size, i.e., $M$ is approximately a constant. Thus, the computational time for evaluating near-field interaction scales linearly with the system size. The farfield potential at $\mathbf{r}$ is decomposed into the contributions from the farfield cubes of different levels. For instance, at the lowest level the contribution to $V_{\text {far }}(\mathbf{r})$ is from those farfield cubes whose parents are considered as near-field; the contribution from the next higher level (parents) is thus from the farfield parent cubes whose grandparents are of near-field, and so on. At a particular level, the multipole expansions of the potentials [cf. Eq. (40)] from the contributing farfield cubes at the same level are converted into local Taylor expansions about the center of the current cube $B$, and the expansion coefficients from different cubes are summed. The Taylor expansion coefficients are computed by expanding each term of Eq. (40) in $\mathbf{r}$ which is the displacement vector from the center of cube $B$, for example,

$$
\frac{Z}{\left|\mathbf{r}-\mathbf{r}_{A B}\right|}=\frac{Z}{r_{A B}}+\frac{Z \mathbf{r}_{A B}}{r_{A B}^{3}} \cdot \mathbf{r}+\cdots
$$

$\frac{\mu \cdot\left(\mathbf{r}-\mathbf{r}_{A B}\right)}{\left|\mathbf{r}-\mathbf{r}_{A B}\right|^{3}}=\frac{-\mu \cdot \mathbf{r}_{A B}}{r_{A B}^{3}}+\left[\frac{\mu}{r_{A B}^{3}}-3 \frac{\left(\mu \cdot \mathbf{r}_{A B}\right) \mathbf{r}_{A B}}{r_{A B}^{5}}\right] \cdot \mathbf{r}+\cdots$.

The above Taylor expansion begins at the top level and then transverse downward. The information of the parent cubes are translated to the children and is continued to the lowest level. $V_{\text {far }}(\mathbf{r})$ of a cube at the lowest level is a Taylor expansion that contains the potential of all induced charge distributions from all the farfield cubes. Given a cube at level $l$, the number of its near-field cubes is $(2 D+1)^{3}$. The number of its farfield cubes whose parents are of the near-field is thus $7 \times(2 D+1)^{3}$, assuming there are $B_{l}$ cubes at level $l$ and the Taylor coefficients are evaluated for every cube. Thus, the CPU cost for calculating these Taylor expansions is proportional to

$$
B_{l}\left[7(2 D+1)^{3}\right]
$$

At the next higher level $l-1$, there are $B_{l-1}=B_{l} / 8$ cubes, and the CPU time to calculate the Taylor expansions for these cubes is thus proportional to 


$$
\frac{7 B_{l}}{8}\left[(2 D+1)^{3}\right] .
$$

Therefore, the total CPU time for calculating the Taylor expansions is proportional to

$$
\begin{aligned}
& B_{l}\left[7 \times(2 D+1)^{3}\right]+B_{l-1}\left[7 \times(2 D+1)^{3}\right] \\
&+ B_{l-2}\left[7 \times(2 D+1)^{3}\right]+\cdots \\
&= B_{\text {lowest }}\left[7 \times(2 D+1)^{3}\right]+\frac{B_{\text {lowest }}}{8}\left[7 \times(2 D+1)^{3}\right] \\
& \quad+\frac{B_{\text {lowest }}}{8^{2}}\left[7 \times(2 D+1)^{3}\right]+\cdots \\
& \leqslant 8 B_{\text {lowest }}(2 D+1)^{3},
\end{aligned}
$$

where $B_{\text {lowest }}$ is the number of cubes in the lowest level. Since $B_{\text {lowest }}$ increases linearly with the system size, the computational time for evaluating $V_{\text {far }}$ for all the cubes at the lowest level scales also linearly with the system size.

For $V_{i j m n}^{\mathrm{xc}}$, we resort to numerical integration to calculate it since the analytical results cannot be obtained, even using Gaussian orbitals. In order to achieve the high accuracy, sophisticated multicenter quadrature schemes ${ }^{27,28,42}$ are used. The integrals are partitioned over atomic centers using a weight scheme, and a further decomposition into radial and angular components of each atomic contribution is introduced. ${ }^{42-44}$ Since the number of grid points is proportional to the size of the molecule, and we have to calculate the integrals over four orbital indices, the numerical integration of $V_{i j m n}^{\mathrm{xc}}$ is an $O\left(N^{5}\right)$ computational process. Taking advantage of the fast decaying nature of Gaussian basis functions, there are only a limited number of basis functions with nonnegligible value at a given grid point. The computational time is therefore proportional to the number of grid points. In addition, we discard the integrals when the differential overlap between any two orbitals is negligible, i.e.,

$V_{i j m n}^{\mathrm{xc}}=0, \quad$ if $S_{k l}<1 \times 10^{-14}$,

where $k$, or $l=i, j, m$, or $n$.

This results in an $O(N)$ computational time for evaluating all $V_{i j m n}^{\mathrm{xc}}$. To further reduce the computational time, we exploited the locality of the exchange-correlation potential and confine its contribution at a given grid point to a relatively small region around it with a negligible loss of accuracy.

In Eq. (23) the summation over $k$ is restricted to a finite range, which does not depend on the value of $N$ when the size is large enough. Since the number of non-negligible $\delta \rho_{i j}^{(1)}$ is proportional to $N$, the total number of steps required to integrate scales linearly with $N$. Therefore, the overall computational time is proportional to $N$.

\section{Transformation between atomic orbital basis set and orthogonal atomic orbital basis set}

The LDM method was originally developed for semiempirical models which adopt orthogonal basis sets. Firstprinciples methods employ the localized Gaussian basis sets whose orbitals are nonorthogonal. In Ref. 39, we developed

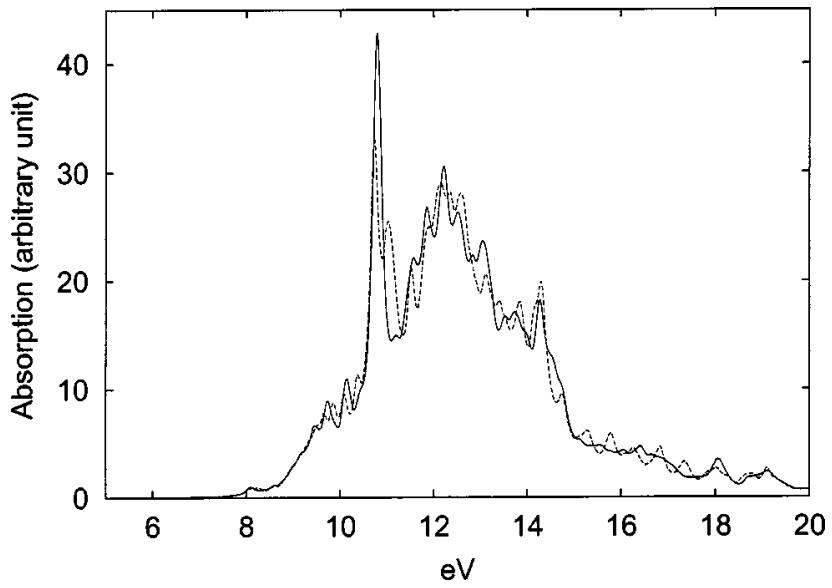

FIG. 5. Absorption spectra for $\mathrm{C}_{40} \mathrm{H}_{82}$. The solid line is for $\mathrm{C}_{40} \mathrm{H}_{82}$ and $l$ $=25 \AA$, and the dashed line for the full TDDFT calculation, where no cutoffs are introduced. The phenomenological dephasing constant $\Gamma=0.1 \mathrm{eV}$.

a generalized LDM (GLDM) method that adopts nonorthogonal basis functions. The GLDM method was found sensitive to the cutoffs and sometimes divergent during the numerical simulations. We start from Gaussian AOs. Each of these orbitals is localized at a particular atom. However, they are not orthogonal. To implement TDDFT with the LDM method, we need to orthogonalize the AOs while maintaining the localized nature of the basis functions. Cholesky transformation has been used to orthogonalize AOs and it has been found that the resulting orthonormal AOs are quite localized in space. ${ }^{20}$ Subsequently, both the density and Fock matrices are evaluated in terms of the orthonormal basis set, and the EOMs [Eqs. (21)-(23)] need no modifications. Specifically, the transformation is based on the Cholesky decomposition of the overlap matrix $S$ to orthogonalize the atomic orbitals (AOs). The Cholesky decomposition partitions a positive definite matrix into a lower triangular matrix times an upper triangular matrix, ${ }^{45}$

$$
S=U^{T} U,
$$

where $U$ is an upper triangular matrix. For symmetric positive definite $S$, the lower triangular matrix is just the transpose of the upper triangular matrix, and the Cholesky transformation to an orthonormal basis can be written as

$$
\rho^{\text {ortho }}=U \rho^{\mathrm{AO}} U^{T} ; \quad h^{\text {ortho }}=U^{-T} h^{\mathrm{AO}} U^{-1} .
$$

The transformed reduced density matrix is found to have the same sparsity as the reduced density matrix in an AO basis, and the transformed Fock matrix has similar sparsity as the reduced density matrix.

The computational cost of Cholesky factorization [Eq. (48)] is given by ${ }^{20}$

$$
\mathrm{CPU} \text { time } \propto \sum_{i=1}^{N} m_{i}^{U}\left(m_{i}^{U}+3\right) / 2,
$$

where $m_{i}^{U}$ is the number of nonzero elements in the $i$ th row of $U$ and $N$ is the number of basis functions. Taking advantage of the sparsity of overlap matrix $S, m_{i}^{U}$ remains approxi- 


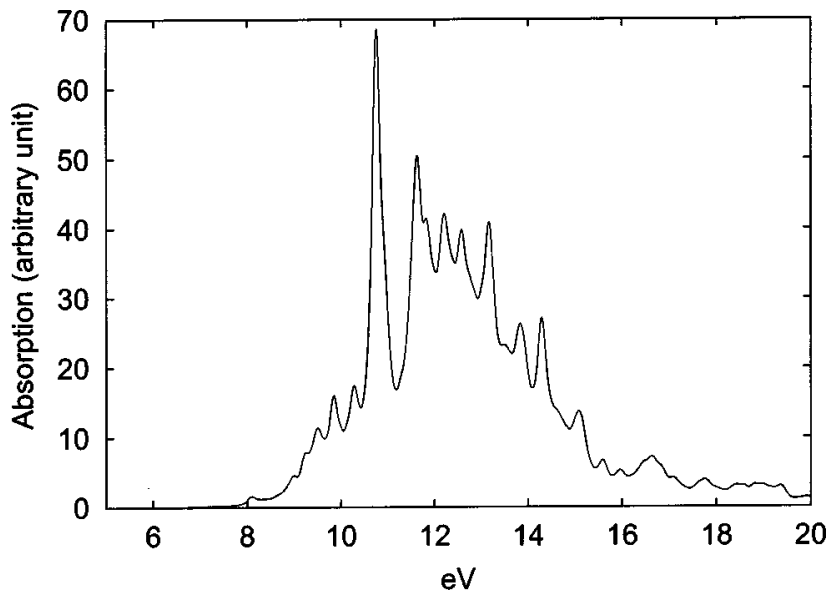

FIG. 6. Absorption spectra for $\mathrm{C}_{60} \mathrm{H}_{122}$ using $l_{0}=l_{1}=25 \AA$. The phenomenological dephasing constant $\Gamma=0.1 \mathrm{eV}$.

mately constant regardless of the system size. Similar results hold for the computation of inverse Cholesky matrix. The CPU time thus goes up linearly with $N$.

\section{RESULTS}

\section{A. Linear-scaling calculation on linear alkanes}

To demonstrate that the TDLDA-LDM method is indeed a linear-scaling method, we have carried out calculations on a series of linear alkanes. Gaussian basis set 6-31G has been used. We apply the electric field $\mathcal{E}(t)$ parallel to the linear alkanes. The time step of the simulation is set to $0.005 \mathrm{fs}$ and the total simulation time is $70 \mathrm{fs}$. The accuracy of the calculation is determined by the values of $l_{0}$ and $l_{1}$. For simplicity, we chose $l_{0}=l_{1}=l$ in our calculation. In Fig. 5, we present the absorption spectrum of $\mathrm{C}_{40} \mathrm{H}_{82}$ using $l=25 \AA$. To examine the accuracy of the calculation, we perform a full TDDFT calculation with no cutoff for the same molecule. The dashed line represents the results of the full TDLDA calculation. The agreement between the results of two TDLDA calculations are excellent. This value $l=25 \AA$ will be employed in the calculation of larger alkanes. In Fig. 6,

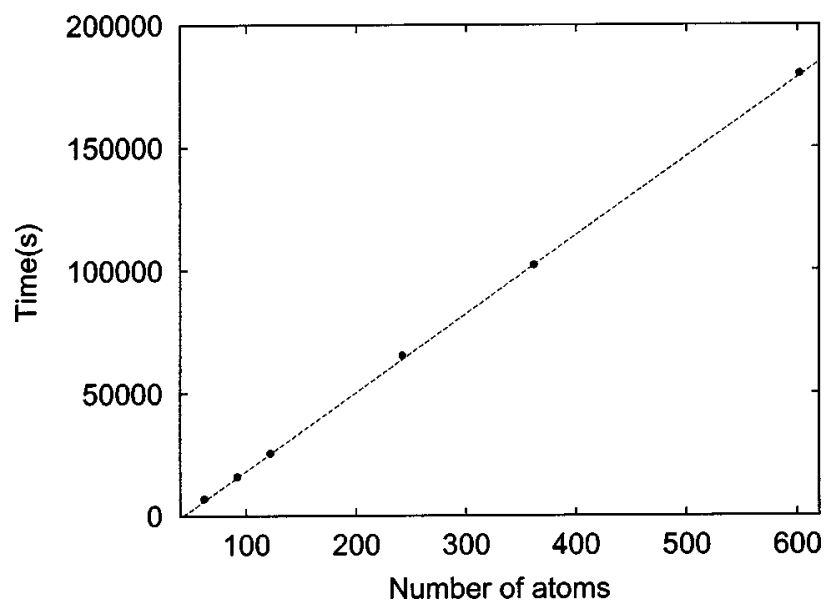

FIG. 7. CPU time for alkanes with $N=62,92,122,242,362$, 602. Each calculation is performed during the time interval between -0.5 and $0.5 \mathrm{fs}$ with time step 0.005 fs. $l=25 \AA$ is used.

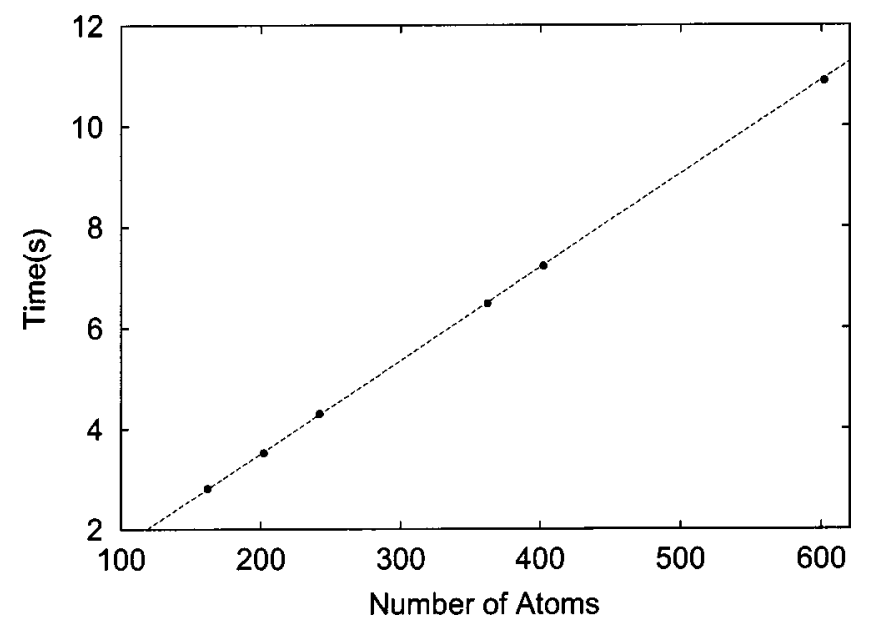

FIG. 8. CPU time for the Cholesky transformation.

we plot the absorption spectrum of $\mathrm{C}_{60} \mathrm{H}_{122}$. In Figs. 5 and 6, absorptions start at about $8 \mathrm{eV}$ for both molecules. This is consistent with the observed $\sigma$ to $\sigma^{*}$ transition at about 150 $\mathrm{nm}$ wavelength in alkanes. ${ }^{46}$ The absorption spectrum of $\mathrm{C}_{40} \mathrm{H}_{82}$ and $\mathrm{C}_{60} \mathrm{H}_{122}$ are overall similar, except for the slight difference between 11 and $13 \mathrm{eV}$.

Now we are ready to examine whether the TDLDALDM method is indeed a linear-scaling method. We perform the TDLDA-LDM calculates on a series of linear alkanes with the number of atoms ranging from 62 to 602 . To save computational time, we limit our simulation to a $1 \mathrm{fs}$ interval. The CPU time of each calculation is recorded. The computational time spent in solving the DFT ground state is negligible. In Fig. 7, we plot the CPU time versus $N$. Clearly the CPU time scales linearly with $N$ for $N$ between 62 and 602 . The linear scaling of computational time versus the system size has been convincingly achieved. We have also examined the CPU time of Cholesky transformation versus the system size. Figure 8 shows the computational time of Cholesky transformation scales linearly with the molecular size.

\section{B. TDDFT calculations on polyacetylene oligomers}

We have also performed the TDLDA-LDM calculations on several polyacetylene oligomers. Their excitation energies are presented in Table I. A 6-31G basis set is employed and no cutoff is used in the calculations. $\mathcal{E}(t)$ is set parallel to the molecules. The time step and total time of the simulation are 0.005 and $70 \mathrm{fs}$, respectively. We study the excitation to the optically allowed $1{ }^{1} B_{u}$ state. Compared with the available experimental excitation energies for the oligomers, it is con-

TABLE I. Excitation energies (eV) of polyacetylene oligomers.

\begin{tabular}{rcc}
\hline \hline$N$ & TDLDA & Expt. \\
\hline 2 & 8.20 & 7.65 (Ref. 50) \\
4 & 6.01 & 5.92 (Ref. 50) \\
6 & 4.70 & 4.95 (Ref. 50) \\
8 & 4.22 & 4.41 (Ref. 50) \\
10 & 3.98 & 4.02 (Ref. 50) \\
20 & 2.25 & 2.80 (Ref. 51) \\
\hline \hline
\end{tabular}




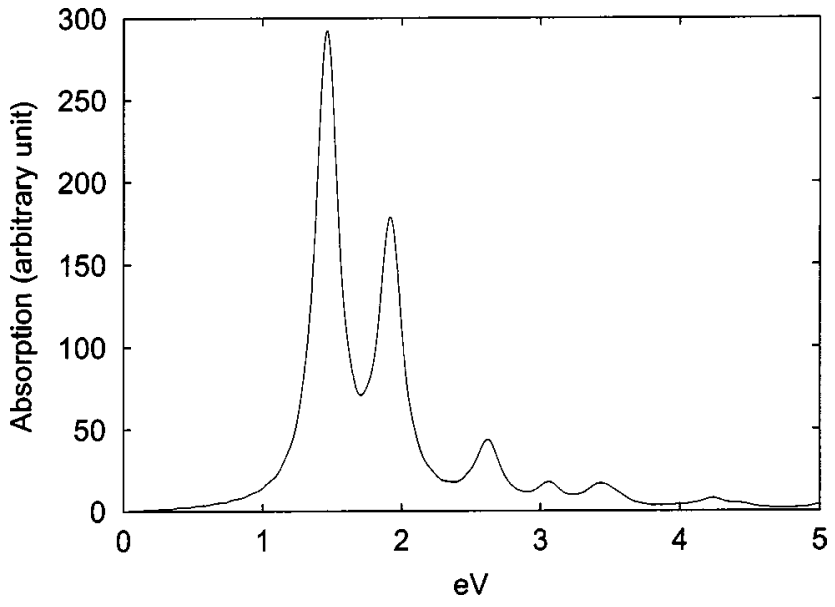

FIG. 9. Absorption spectra for $\mathrm{C}_{40} \mathrm{H}_{42}$.

firmed that our TDLDA-LDM code yields reasonable predictions for excitation energies. The general trend of decreasing excitation energy with the chain length is correctly reproduced. For molecule $\mathrm{C}_{20} \mathrm{H}_{22}$, the calculated excitation energy is less than the experiment result. This may be due to the structural difference between the experiment and calculation. Long polymers tend to bend or rotate compared to the optimized structure adopted in the calculations, which leads to the reduction of conjugation length and the blue shifts of absorption spectra. We calculate the absorption spectra of longer oligomers, such as $\mathrm{C}_{40} \mathrm{H}_{42}$, whose absorption spectrum is given in Fig. 9. The optical gap of longer oligomers is believed to saturate at about $2 \mathrm{eV}$, which contrasts our calculation result for $\mathrm{C}_{40} \mathrm{H}_{42}$. This may again be due to the structural distortions of long polyacetylene oligomers in experiments. Of course, this may also imply that the TDLDA calculation is not adequate for longer polyacetylene oligomers. A further study is warranted. It has been pointed out that the excitation energy to the $1{ }^{1} B_{u}$ is sensitive to the choice of XC functional. ${ }^{47}$ It has been found that the current $\mathrm{XC}$ functionals are inadequate to describe the excitation energies, polarizabilities, and hyperpolarizabilities of polyacetylene ${ }^{48,49}$ Better XC functionals beyond the existing $\mathrm{XC}$ functionals are required for calculating the excited state properties of polyacetylene and other systems. Since the LDM method is based solely on the locality of the reduced density matrix, we can thus improve our TDDFT-LDM method by introducing more sophisticated exchangecorrelation functionals.

\section{DISCUSSION AND CONCLUSION}

The integration of Eq. (23) alone does not yield linear scaling. It requires matrix multiplications that scale as $O\left(N^{3}\right)$. For large molecular systems, we can reduce the cost of the matrix operations by taking advantage of matrix sparsity. The intrinsic nature and size of the molecular system under consideration are important factors in determining the sparsity. It is well known that systems with a smaller HOMO-LUMO gap have denser Fock and density matrices than those with a large HOMO-LUMO gap. ${ }^{20,21}$ In the large molecule limit, the number of significant elements of a den- sity matrix are proportional to the molecular size because of its localized nature. One should also notice that DFT Fock and density matrices are normally much less sparse than the semiempirical ones. In order to attain the same accuracy, longer cutoff length $l$ should be chosen compared to that of semiempirical methods. ${ }^{35,36,52,53}$ The only approximation made here relates to the feature of a reduced single-electron density matrix, i.e., the locality of $\rho$ and $\delta \rho$. This fact guarantees the wide applicability of the method. To improve our TDDFT-LDM method, we may adopt other XC functionals, such as, GGA ${ }^{54-57}$ and B3LYP. ${ }^{58}$ We may also improve our FMM by the $\mathrm{CFMM}^{25}$ or $\mathrm{GvFMM}^{26}$ approach. The current bottleneck of the TDLDA-LDM method is the evaluation of $\delta h^{u}$ at each simulation step. We may update the farfield part of $\delta h^{u}$ every few simulation steps. Another issue is the stability of the simulation. The numerical simulations may sometimes diverge, caused by an unphysical numerical fluctuation, leading to a positive feedback for $\delta \rho^{(1)}$. We may adopt a purification procedure to suppress the unphysical numerical fluctuation.

To summarize, we have developed a linear-scaling TDLDA-LDM method. The calculations on the linear alkanes and polyacetylene oligomers demonstrate its accuracy and efficiency. This makes possible the first-principles calculation of the excited state properties of very large molecular systems. Although the linear response has been the focus, a nonlinear response may easily be evaluated via a slight generalization of the method.

\section{ACKNOWLEDGMENTS}

Support from the Hong Kong Research Grant Council (RGC) and the Committee for Research and Conference Grants (CRCG) of the University of Hong Kong is gratefully acknowledged.

${ }^{1}$ P. Hohenberg and W. Kohn, Phys. Rev. 136, B864 (1964).

${ }^{2}$ W. Kohn and L. J. Sham, Phys. Rev. 140, A1133 (1965).

${ }^{3}$ R. G. Parr and W. Yang, in Density-Functional Theory of Atoms and Molecules (Oxford University Press, Oxford, 1989).

${ }^{4}$ J. A. Pople, R. Seeger, and R. Krishnan, Int. J. Quantum Chem., Symp. 11, 149 (1977).

${ }^{5}$ C. Møller and M. S. Plesset, Phys. Rev. 46, 618 (1934).

${ }^{6}$ J. A. Pople, R. Krishnan, H. B. Schlegel, and J. S. Binkley, Int. J. Quantum Chem. 14, 545 (1978).

${ }^{7}$ E. Runge and E. K. U. Gross, Phys. Rev. Lett. 52, 997 (1984).

${ }^{8}$ M. J. Stott and E. Zaremba, Phys. Rev. A 21, 12 (1980).

${ }^{9}$ A. Zangwill and P. Soven, Phys. Rev. Lett. 45, 204 (1980).

${ }^{10}$ G. D. Mahan, Phys. Rev. A 22, 1780 (1980).

${ }^{11}$ M. E. Casida, in Recent Developments and Applications of Modern Density functional Theory, Theoretical and Computational Chemistry, edited by J. M. Seminario (Elsevier Science, Amsterdam, 1996), Vol. 4.

${ }^{12}$ S. M. Colwell, N. C. Handy, and A. M. Lee, Phys. Rev. A 53, 1316 (1996).

${ }^{13}$ V. Chernyak and S. Mukamel, J. Chem. Phys. 112, 3572 (2000).

${ }^{14}$ S. J. A. van Gisbergen, J. G. Snijders, and E. J. Baerends, J. Chem. Phys. 103, 9347 (1995).

${ }^{15}$ S. Goedecker, Rev. Mod. Phys. 71, 1085 (1999).

${ }^{16}$ W. Yang, Phys. Rev. Lett. 66, 1438 (1991).

${ }^{17}$ W. Yang and T. S. Lee, J. Chem. Phys. 103, 5674 (1995).

${ }^{18}$ W. Kohn, Chem. Phys. Lett. 208, 167 (1993).

${ }^{19}$ W. Kohn, Phys. Rev. Lett. 76, 3168 (1996).

${ }^{20}$ J. M. Millam and G. E. Scuseria, J. Chem. Phys. 106, 5569 (1997).

${ }^{21}$ G. E. Scuseria, J. Phys. Chem. A 103, 4782 (1999).

${ }^{22}$ C. F. Guerra, J. G. Snijders, G. te Velde, and E. J. Baerends, Theor. Chem. Acc. 99, 391 (1998)

${ }^{23}$ L. Greengard, Science 265, 909 (1994). 
${ }^{24}$ H. Q. Ding, N. Karasawa, and W. A. Goddard III, J. Chem. Phys. 97, 4309 (1992).

${ }^{25}$ C. A. White, B. G. Johnson, P. M. W. Gill, and M. Head-Gordon, Chem. Phys. Lett. 253, 268 (1996); C. A. White, B. G. Johnson, P. M. W. Gill, and M. Head-Gordon, ibid. 230, 8 (1994).

${ }^{26}$ M. C. Strain, G. E. Scuseria, and M. J. Frisch, Science 271, 51 (1996).

${ }^{27}$ R. E. Stratmann, G. E. Scuseria, and M. J. Frisch, Chem. Phys. Lett. 257, 213 (1996).

${ }^{28}$ J. M. Pérez-Jordá and W. Yang, Chem. Phys. Lett. 241, 469 (1995).

${ }^{29}$ X. P. Li, R. W. Nunes, and D. Vanderbilt, Phys. Rev. B 47, 10891 (1993).

${ }^{30}$ S. Goedecker and L. Colombo, Phys. Rev. Lett. 73, 122 (1994); S. Goedecker and M. Teter, Phys. Rev. B 51, 9455 (1995).

${ }^{31}$ F. Mauri, G. Galli, and R. Car, Phys. Rev. B 47, 9973 (1993).

${ }^{32}$ P. Ordejón, D. A. Drabold, M. P. Grumbach, and R. M. Martin, Phys. Rev. B 48, 14646 (1993).

${ }^{33}$ A. Takahashi and S. Mukamel, J. Chem. Phys. 100, 2366 (1994).

${ }^{34}$ G. H. Chen and S. Mukamel, J. Am. Chem. Soc. 117, 4945 (1995).

${ }^{35}$ S. Yokojima and G. H. Chen, Phys. Rev. B 59, 7259 (1999).

${ }^{36}$ S. Yokojima and G. H. Chen, Chem. Phys. Lett. 292, 379 (1998).

${ }^{37}$ E. R. Davidson, J. Comput. Phys. 17, 87 (1975).

${ }^{38}$ G. H. Chen and S. Mukamel, J. Phys. Chem. 100, 11080 (1996).

${ }^{39}$ W. Z. Liang, S. Yokojima, and G. H. Chen, J. Chem. Phys. 110, 1844 (1999).

${ }^{40}$ E. K. U. Gross and W. Kohn, in Advances in Quantum Chemistry, edited by S. B. Trickey (Academic, San Diego, 1990).

${ }^{41}$ A. Szabo and N. S. Ostlund, in Modern Quantum Chemistry: Introduction to Advanced Electronic Structure Theory (Dover, New York, 1996).

${ }^{42}$ A. D. Becke, J. Chem. Phys. 88, 2547 (1988).

${ }^{43}$ Handbook of Mathematical Functions, edited by M. Abramowitz and I. A. Stegun (Dover, New York, 1970).
${ }^{44}$ V. I. Lebedev, Proceedings of the Conferences in Novosibirsk 1978, edited by S. L. Sobolev (Nauka Sibirsk. Otdel., Novosibirsk, 1980).

${ }^{45}$ W. H. Press, S. A. Teukolsky, W. T. Vetterling, and B. P. Flannery, Numerical Recipes in FORTRAN, 2nd ed. (Cambridge University Press, New York, 1992).

${ }^{46}$ W. Kemp, in Organic Spectroscopy (Macmillan, London, 1991).

${ }^{47}$ C. P. Hsu, S. Hirata, and M. Head-Gordon, J. Phys. Chem. 105, 451 (2001).

${ }^{48}$ S. Hirata, M. Head-Gordon, and R. J. Bartlett, J. Chem. Phys. 111, 10774 (1999).

${ }^{49}$ B. Champagne, E. A. Perpete, S. J. A. van Gisbergen, E. J. Baerends, J. G. Snijders, C. Soubra-Ghaoui, K. A. Robins, and B. Kirtman, J. Chem. Phys. 109, 10489 (1998).

${ }^{50}$ J. Ma, S. Li, and Y. Jiang, Macromolecules 35, 1109 (2002).

${ }^{51}$ J. L. Bredas, R. Silbey, D. S. Boudreaux, and R. R. Chance, J. Am. Chem. Soc. 105, 6555 (1983).

${ }^{52}$ S. Yokojima, D. H. Zhou, and G. H. Chen, Chem. Phys. Lett. 333, 397 (2001).

${ }^{53}$ W. Z. Liang, X. J. Wang, S. Yokojima, and G. H. Chen, J. Am. Chem. Soc. 122, 11129 (2000); W. Z. Liang, G. H. Chen, Z. M. Li, and Z. K. Tang, Appl. Phys. Lett. 80, 3415 (2002).

${ }^{54}$ A. D. Becke, Phys. Rev. A 38, 3098 (1988).

${ }^{55}$ C. Lee, W. Yang, and R. G. Parr, Phys. Rev. B 37, 785 (1988).

${ }^{56}$ J. P. Perdew, K. Burke, and M. Ernzerhof, Phys. Rev. Lett. 77, 3865 (1996).

${ }^{57}$ J. P. Perdew, K. Burke, and Y. Wang, Phys. Rev. B 54, 16533 (1996).

${ }^{58}$ A. D. Becke, J. Chem. Phys. 98, 5648 (1993). 